\title{
Investigating lower stratospheric model transport: Lagrangian calculations of mean age and age spectra in the GCM ECHAM4
}

\author{
Christian Reithmeier • Robert Sausen • \\ Volker Grewe
}

Received: 12 December 2005/ Accepted: 26 June 2007/Published online: 27 July 2007

(C) Springer-Verlag 2007

\begin{abstract}
The Lagrangian scheme ATTILA is used to calculate age spectra and the mean age of air in the general circulation model ECHAM4. The advantage of the Lagrangian method is that temporal variation in transport is taken into account and that beyond transport times the actual transport pathways can be investigated. We found a strong seasonal cycle in mean age and age spectra, especially at high latitudes. When plotting polar age spectra against time, it can clearly be seen that the edge of the polar vortex acts as an efficient transport barrier and that exchange with extra-polar air takes place only for a short period of approximately two months after the polar vortex has broken down. Compared to observations the mean age is reproduced satisfactorily below approximately $20 \mathrm{~km}$. Above that level however, the mean age is underestimated, especially at high latitudes. Furthermore, the observed sharp meridional gradient is located too far polewards in the model, which indicates that the subtropical transport barrier is too weak. There is a distinct variation in the shape of the age spectra with latitude. At low latitudes the age spectra consist of one single peak, whereas at higher latitudes secondary peaks appear, which are one year apart and whose positions in the spectrum are independent of the location. At polar latitudes there are even several peaks of
\end{abstract}

C. Reithmeier · R. Sausen $(\bowtie) \cdot$ V. Grewe

Institut für Physik der Atmosphäre,

Deutsches Zentrum für Luft- und Raumfahrt (DLR) e.V.,

82234 Wessling, Germany

e-mail: robert.sausen@dlr.de

V. Grewe

e-mail: volker.grewe@dlr.de

Present Address:

C. Reithmeier

BMW Group, 80788 München, Germany approximately equal size. We explain these peaks with two superposing processes. First, the seasonal cycle of the upward mass flux at the tropical tropopause produces a single peak age distribution. And second, at polar latitudes, the temporal evolution of the polar vortex allows mixing of polar and subtropical air only once a year, which results in a superposition of these single peak age distributions. A final investigation of the transport pathways gave indications for predominant routes from the tropics to high latitudes resulting in altitude dependent meridional transport, however, more detailed studies of 3D trajectory data will be needed to clarify this issue.

\section{Introduction}

Knowledge of stratospheric transport processes is an important key when assessing the impact of pollutants on the chemical composition of the stratosphere. It is generally agreed that air enters the stratosphere in the tropics and then moves upward and to higher latitudes as described by the Brewer-Dobson circulation. In addition, transport is influenced by waves propagating upward from the troposphere and breaking in the stratosphere (e.g., Holton et al. 1995).

The mixing ratio of any species in the stratosphere is then determined by the transport from source regions and the chemistry (which may act both as source and sink) along the transport pathways. This combined effect of transport and chemistry makes it particularly difficult to draw conclusions on the transport from observations of reactive tracers. However, although the mixing ratio of a tracer is the key input for assessing the impact of perturbations on the climate system, transport and chemistry are 
two different aspects which have to be investigated separately in order to get a profound knowledge of those processes determining the distribution of a tracer.

Insight into stratospheric transport processes can be gained by measurements of inert tracers with a known time-dependent boundary condition, i.e., tracers whose mixing ratios at the tropical tropopause are known. In that case, the mean transit time from the tropical tropopause to a point $\vec{r}$ in the stratosphere can be inferred from tracer measurements at $\vec{r}$. This transit time is commonly called the mean age $\Gamma(\vec{r})$ of air at $\vec{r}$, and provides a powerful transport diagnostic independent of chemistry.

However, since an air parcel is distorted and mixed with ambient air during its course through the stratosphere, an air sample at $\vec{r}$ cannot be characterized by a single transit time, but it consists of many "irreducible parcels" all having a different transport history. Thus, it is more appropriate to characterize the air at $\vec{r}$ by a distribution of transit times, which is called the age spectrum at $\vec{r}$. This was recognized by Kida (1983), and Hall and Plumb (1994) laid down the theoretical framework for this concept (for an overview see Waugh and Hall 2002). In their notation the age spectrum is written as $G\left(\vec{r}, \mathcal{R}, t^{\prime}\right)$, which means that $G\left(\vec{r}, \mathcal{R}, t^{\prime}\right) d t^{\prime}$ is the mass fraction of "irreducible parcels" in an air sample at $\vec{r}$ which needed a time $\tilde{t} \in\left[t^{\prime} ; t^{\prime}+d t^{\prime}\right)$ to travel from $\mathcal{R}$ to $\vec{r}$. Here, $\mathcal{R}$ is identified as the tropical tropopause where most air enters the stratosphere.

If the age spectrum is known, the mass mixing ratio $c(\vec{r}, t)$ of an arbitrary inert tracer at some point $\vec{r}$ in the stratosphere can easily be computed from the history of the mass mixing ratio $c\left(\mathcal{R}, t^{\prime}\right), t^{\prime}<t$ at the tropical tropopause

$c(\vec{r}, t)=\int_{-\infty}^{t} c\left(\mathcal{R}, t^{\prime}\right) G\left(\vec{r}, \mathcal{R}, t-t^{\prime}\right) d t^{\prime}$

This formula can be recognized as a mass weighted average whereby parcels which travelled a time $t-t^{\prime}$ (from $\mathcal{R}$ to $\vec{r}$ ) entered the stratosphere at $t^{\prime}$ and thus carry the mass mixing ratio $c\left(\mathcal{R}, t^{\prime}\right)$ which is constant in the stratosphere since the tracer has no sink there by assumption. Similarly, the mean age $\Gamma(\vec{r})$ is readily seen to be the first moment of the age spectrum, i.e., the average of the transit times (see Hall and Plumb 1994, for details):

$\Gamma(\vec{r})=\int_{0}^{\infty} t^{\prime} G\left(\vec{r}, \mathcal{R}, t^{\prime}\right) d t^{\prime}$

However, this theory assumes that stratospheric transport is stationary, i.e., does not change with time. Considering the temporal variation of transport, the age spectrum depends not only on the location $\vec{r}$ but also on the time $t$ and has to be written as $G\left(\vec{r}, \mathcal{R}, t^{\prime}, t\right)$. It is then $G\left(\vec{r}, \mathcal{R}, t^{\prime}, t\right) d t^{\prime}$ the mass fraction in an air sample at $\vec{r}$ and $t$ which needed a time $\tilde{t} \in\left[t^{\prime} ; t^{\prime}+d t^{\prime}\right)$ to travel from $\mathcal{R}$ to $\vec{r}$, and thus entered the stratosphere at $t-t^{\prime}$ and arrived at $\vec{r}$ at $t$.

Most studies neglect the seasonal and interannual variation in the age spectrum, however, in this paper we will present age spectra for different seasons/months and we will show how additional information on stratospheric transport can be gained from this.

Since the age spectrum is not observable (Andrews et al. 1999, 2001, inferred the age spectrum from observations, but they had to make assumptions on the shape of the spectra), we calculate the age spectrum for the general circulation model ECHAM4. Hence, all presented results refer to the stratospheric transport in this model.

There are two methods to calculate age spectra. The first one is an Eulerian calculation. An inert tracer with an e.g. Dirac- $\delta$ like boundary condition is calculated, i.e., a tracer pulse is released at the tropopause. The time series of the tracer mixing ratio at a given point in the stratosphere gives the age spectrum at this point. Falling back on the theory of Hall and Plumb (1994), such a calculation means the calculation of the Green function solution of the model transport operator, which is assumed to be stationary. If the tracer pulse is released at time $t_{0}$, the boundary condition can be written as $c(\mathcal{R}, t)=c_{0} \delta\left(t-t_{0}\right)$, where $c_{0}$ is an arbitrary initial mixing ratio, and the above formula gives the (non-normalized) age spectrum by

$c(\vec{r}, t)=\int_{-\infty}^{t} c_{0} \delta\left(t^{\prime}-t_{0}\right) G\left(\vec{r}, \mathcal{R}, t-t^{\prime}\right) d t^{\prime}=c_{0} G\left(\vec{r}, \mathcal{R}, t-t_{0}\right)$.

However, if non-stationarity is assumed, the temporal evolution of the mass mixing ratio equals

$$
\begin{aligned}
c(\vec{r}, t) & =\int_{-\infty}^{t} c_{0} \delta\left(t^{\prime}-t_{0}\right) G\left(\vec{r}, \mathcal{R}, t-t^{\prime}, t\right) d t^{\prime} \\
& =c_{0} G\left(\vec{r}, \mathcal{R}, t-t_{0}, t\right)
\end{aligned}
$$

and gives only one value $\left(t-t_{0}\right)$ of the age spectrum at $\vec{r}$ and $t$. Thus, in order to calculate the age spectrum for a fixed time $t$, many tracer experiments with different times $t_{0}$ of the tracer pulse have to be conducted. Furthermore, if the transport operator varies also on interannual scale and one wants to calculate a climatological mean of the age spectrum over several $t+n$ years, $n=0,1,2, \ldots$, even more tracers are needed.

The second method is a Lagrangian calculation. Many trajectories are calculated in the stratosphere and after a long time ( $\sim 10$ years) the age spectrum at some point $(\vec{r}, t)$ is simply calculated by sampling the trajectories around 
that point with respect to their transit time since entering the stratosphere. An advantage of this method is that it is straightforward and that no assumptions on the stationarity of the transport have to be made, i.e., choosing different times $t$ results in different age spectra. A further advantage is that Lagrangian transport is numerically non-diffusive. Eluszkiewicz et al. (2000) showed that the mean age strongly depends on the transport scheme used, and that numerically diffusive schemes produce the most unrealistic results. A disadvantage of the Lagrangian method is that a large number of trajectories have to be computed, because (1) the trajectory density, which is proportional to the air density, decreases exponentially with height, and (2) the number of trajectories has to be large enough to produce statistically stable results (cf. Schoeberl et al. 2000). On the other hand, the Lagrangian method takes into account the non-stationarity of transport, reducing the computational costs compared to the Eulerian calculation (see above).

In this paper, we use the recently developed Lagrangian transport scheme ATTILA (Reithmeier and Sausen 2002), which has also been applied in Lelieveld et al. (2006), Stenke et al. (2007), and Grewe et al. (2007) to calculate the mean age and the age spectrum in ECHAM4. The main goal of this paper is to derive new information from these calculations on the stratospheric transport in ECHAM4. The results regarding mean age will be compared to other models and observations reported in the "Models and Measurements Intercomparison II" report (Park et al. 1999; Hall et al. 1999). However, the focus of this paper is on the seasonal variation of the age spectrum, since to the author's knowledge only limited studies exist. E.g. a number of studies concentrated on the pathways from the troposphere to the stratosphere in order to assess the water vapour mixing ratios entering the stratosphere, based on trajectory calculations (Hatsushika and Yamazaki 2003; Bonazzola and Haynes 2004; Fueglistaler et al. 2004, 2005; Fueglistaler and Haynes 2005). Fueglistaler et al. (2005) showed that minimum water vapour mixing ratios occurring during boreal winter are well explained by low cold point temperatures, which result from enhanced tropical ascent. Schoeberl et al. (2003, 2005) investigated stratospheric age spectra and found secondary peaks at mid latitudes, which they associated to two high probability pathways, which is supported by observational evidence (Andrews et al. 2001). Although the age spectrum differs significantly in those studies.

In this paper, we will show how the seasonal variations in the age spectra give information on the dynamics of the polar stratosphere. Since age spectra contain information on the transit times only but not on the actual transport pathways, we will also address the problem of interpreting age spectra correctly, and supplement the results with a trajectory diagnostics to learn more about the transport from the tropics to polar latitudes.

\section{Model description and experimental setup}

The spectral atmospheric general circulation model ECHAM4 is the fourth generation of the Hamburg climate model (Roeckner et al. 1996). It integrates the primitive equations. Prognostic variables are vorticity, divergence, temperature, (logarithm of) surface pressure, humidity, cloud water content (ice and water phase), and, optionally, up to 21 passive tracers.

Humidity, cloud water and the tracers are advected by a semi-Lagrangian transport scheme employed in the grid point domain (Williamson and Rasch 1994). Since this scheme is not mass conserving, a mass fixer is used (Rasch and Williamson 1990). The semi-Lagrangian scheme is used in order to avoid negative concentrations resulting from sharp gradients due to the Gibbs phenomenon, which describes overshoots of the fourrier series of a periodic function at a jump discontinuity. The remaining prognostic variables are advected by a spectral advection scheme.

ECHAM4 contains parameterizations of radiation, cloud formation and precipitation, convection, horizontal diffusion, surface fluxes and vertical diffusion, gravity wave drag, and land surface processes. We apply a slightly modified version of the operational ECHAM4 model in which the numerical formulation of the convection scheme has been altered in order to make it strictly positive (Brinkop and Sausen 1997).

The model atmosphere is vertically partitioned into 19 non-equidistant layers using a hybrid $\sigma-p$-coordinate system (Roeckner et al. 1992). The uppermost model layer is centred at $10 \mathrm{hPa}$, the lowermost layer approximately at $30 \mathrm{~m}$ above the surface. The boundary layer is resolved by approximately 4-5 model layers. In the tropopause region the vertical resolution is about $2 \mathrm{~km}$. A spectral horizontal resolution of T30 was chosen for the current study, corresponding to an isotropic resolution of $6^{\circ}$ on a great circle $(\approx 670 \mathrm{~km})$ for dynamic processes. The associated Gaussian transform grid, which is used for calculating the non-linear terms, diabatic processes, and the semiLagrangian transport, has a resolution of approximately $3.75^{\circ} \times 3.75^{\circ}$. The time step is $30 \mathrm{~min}$.

Atmospheric Tracer Transport In a LAgrangian model (ATTILA) is a Lagrangian transport scheme which runs online within ECHAM4 (Reithmeier and Sausen 2002). It can be used as an alternative to the operational semi-Lagrangian scheme to transport passive tracers. However, in this study ATTILA is not used to transport tracers, but the calculated trajectories are evaluated in order to determine the age of stratospheric air. Therefore, descriptions of the parameterizations in ATTILA specific for tracer transport are not given here, details can be found in Reithmeier (2001) or Reithmeier and Sausen (2002). 
At the chosen horizontal resolution of T30 the model atmosphere is divided into about 190,000 air parcels of constant and equal mass, which move along trajectories, i.e., they are advected using the ECHAM4 wind field. At every ECHAM4 time step the parcel centroids are advected using the fourth order Runge-Kutta method (Press et al. 1990, p. 550). Trajectories are calculated three-dimensionally, i.e., the actual model 3D winds, which include transport due to diabatic heating, are used at parcel locations instead of making isentropic or other assumptions, which would have an impact on the calculation of the mean age of air (Schoeberl et al. 2003). Winds are calculated by linear interpolation horizontally and by cubic Hermite interpolation vertically from the wind field on the ECHAM4 grid. After each advection step, every parcel located in the boundary layer is randomly reallocated in the vertical within the boundary layer (cf. Reithmeier and Sausen 2002).

Since in ATTILA the number density of trajectories is proportional to the air density, there are approximately 19,000 trajectories in the stratosphere above $100 \mathrm{hPa}$, which can be used to calculate the age spectrum. A long term run showed that the initial distribution of the trajectories is stable, i.e., there are no regions in the model domain where trajectories accumulate. Therefore, no reinitialization procedure was included in the model.

Note that the experiment has not been specifically designed to calculate stratospheric age spectra like in other studies (e.g., Eluszkiewicz et al. 2000), where trajectories are released at the tropical tropopause and deleted when they reach the troposphere. As mentioned above, the globally distributed trajectories calculated by the standard version of ATTILA are evaluated. Thus, the results presented below are, so to speak, a by-product of a tracer experiment.

In order to facilitate post-processing, each trajectory was furnished with a clock, which was held at zero in the troposphere and thus measured the time elapsed since a trajectory (in the stratosphere) entered the stratosphere. Since the age of air is often given in terms of the transit time since leaving the boundary layer (e.g., Hall et al. 1999; Hall and Waugh 1997), instead of leaving the troposphere, we introduced a second clock which was held at zero in the boundary layer. Thus, it is possible to investigate the transport times from the surface to the tropical tropopause, and to calculate stratospheric distributions of an inert tracer from surface measurements, which are normally more numerous than at the tropical tropopause. Therfore, most results in this paper are presented in terms of the transit time since leaving the boundary layer.

However, when evaluating globally distributed trajectories as described above, there is the problem that the clocks of the trajectories in the stratosphere are not initialized at the beginning of the run, so that a certain spinup time is needed until most trajectories have crossed the tropopause at least once. The results presented in the following are based on a 25 year run, where the first 10 years have been used as spin-up period (after which $99 \%$ of all trajectories were initialized), and the last 15 years have been evaluated.

\section{Results and discussion}

\subsection{Mean age of air}

Figure 1 shows the zonally averaged mean age of air for all seasons. The mean age is given in terms of the mean transit time since leaving the boundary layer, and is thus comparable to the mean age derived from in situ and surface measurements of $\mathrm{SF}_{6}$. The figure showing the mean transit time since leaving the troposphere shows almost exactly the same pattern as Fig. 1 with the absolute values shifted by 3 months (not shown). This means that the spectrum of the transport times from the surface to the tropical tropopause is narrow with a peak around 0.25 years (Fig. 3). This value is smaller than the value of 0.8 years estimated by Volk et al. (1997) from the time lag between globally averaged surface and upper tropospheric mixing ratios of $\mathrm{SF}_{6}$. However, Ray et al. (1999) found from surface and lowermost stratosphere measurements of $\mathrm{CO}_{2}$ and $\mathrm{SF}_{6}$ that surface air may be transported to the lowermost stratosphere in less than 1.5 months ( 0.125 years).

Figure 1 reveals a distinct seasonal variation of the mean age. The meridional gradients on each hemisphere are in general larger in spring than in fall. This can be explained by the formation of the polar vortex during winter and the associated downwelling of old air at high latitudes. It has to be noted that in ECHAM4 the polar vortex persists longer compared to observations (Hein et al. 2001), so that the gradients are larger in spring than in winter. However, this result is in agreement with the findings of Reddmann et al. (2001), who calculated the mean age from $\mathrm{SF}_{6}$ simulations performed with a middle atmosphere model driven by meteorological analyses, and who also found the strongest gradient to occur in the southern hemisphere in September. Since the polar vortex is stronger and less disturbed in the southern hemisphere, the largest gradients are found in southern midlatitudes during spring. This also indicates that the edge of the polar vortex acts as a transport barrier, which will be further investigated in the next section using age spectra.

The mean age of air can also be inferred from measurements of inert tracers like $\mathrm{CO}_{2}$ or $\mathrm{SF}_{6}$ (Fig. 2). Compared to the observations reported in the "Models and Measurements II" (MM2) model intercomparison (Park 

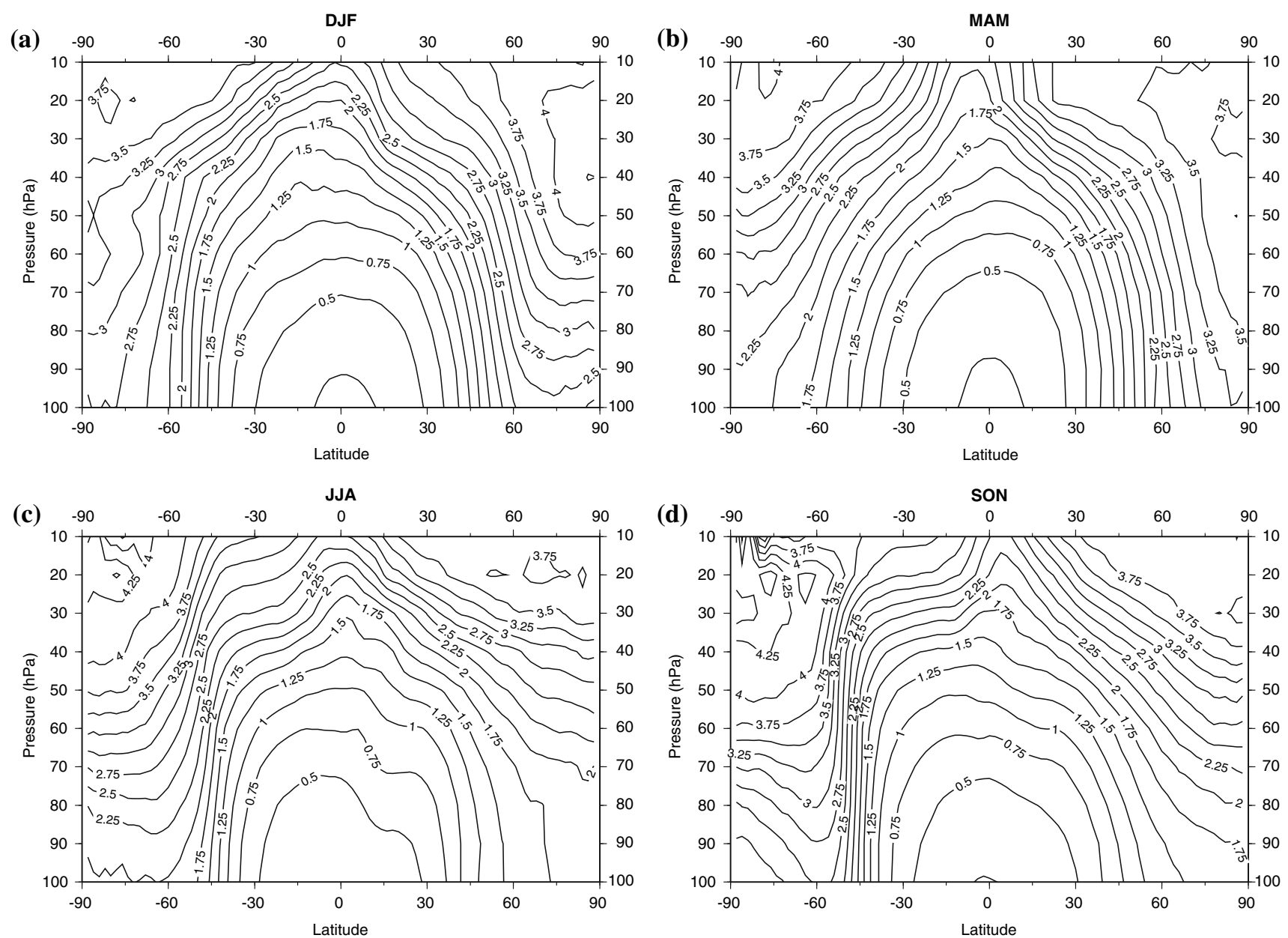

Fig. 1 Mean age of air in spring and fall calculated from the mean transit time of air parcels from the boundary layer. Unit year

et al. 1999; Hall et al. 1999; Waugh and Hall 2002) the mean age of air is generally underestimated in ECHAM4 (ATTILA). This feature is shared with many other models, e.g., virtually all models which participated in MM2 underestimate the mean age (blue shaded area and lines without symbols). At around $20 \mathrm{~km}$ observational data indicate a sharp increase in age from around one year at equatorial regions to around 3.5 years at $30^{\circ}$ of either hemisphere (Fig. 2a). Whereas ATTILA shows a broader area with low values and the sharp increase shifted to higher latitudes, indicating a too weak subtropical barrier. However, clear seasonal differences exist (Fig. 1). This does not imply that there is no sub-tropical barrier, since horizontal distributions of e.g. $\mathrm{N}_{2} \mathrm{O}$ clearly reveal large gradients and horizontal exchange by wave-breaking events (streamer) (Eyring et al. 2003; Grewe 2006). In comparison to the other models within MM2, ATTILA lies at the upper bound of the model range and even surpasses this range at various altitudes and latitudes (Fig. 2b-d). Note that differences may not be explained by different approaches in the determination of the age of air, since the age of air estimates based on $\mathrm{SF}_{6}$ (Fig. 4) and trajectory analysis (Fig. 1) are largely consistent, except for two differences. First, the transport times from the planetary boundary layer to the tropical tropopause are smaller in the $\mathrm{SF}_{6}$ simulation, which is caused by the different methodologies. The trajectory method directly estimates the transport times, whereas the $\mathrm{SF}_{6}$ approach gives estimates based on the concentrations, which is largely dependent on the parameterization of the planetary boundary layer. Secondly, the age of air at polar latitudes above $30 \mathrm{hPa}$ is significantly higher when derived by the $\mathrm{SF}_{6}$ approach. This is likely to be a diagnostic artefact caused by low coverage of the diagnostic grid by Lagrangian particles combined with errors in the methodology, which increase with increasing ages.

At $20 \mathrm{~km}$ altitude (approximately $50 \mathrm{hPa}$ ) the model results agree fairly with observations. Park et al. (1999) report latitudinal mean age variations at $19-20 \mathrm{~km}$ derived from $\mathrm{SF}_{6}$ and $\mathrm{CO}_{2}$ measurements performed during a 3 -week period in October-November 1994. They find a mean age of $0.5-1.0$ years in the tropics, 3-4 years at 
Fig. 2 Comparison of observed (red curves with symbols) and modelled (blue shaded area and curves without symbols) mean age. ATTILA results are indicated by heavy lines, other models results were achieved within the "Model and Measurements II (MM2)" study. Figure adapted from Waugh and Hall (2002); see also Hall et al. (1999) for details. a Latitudinal age distribution at $20 \mathrm{~km}$ height. b-d Vertical age profile at $5^{\circ} \mathrm{S}$, $40^{\circ} \mathrm{N}$, and $65^{\circ} \mathrm{N}$, respectively
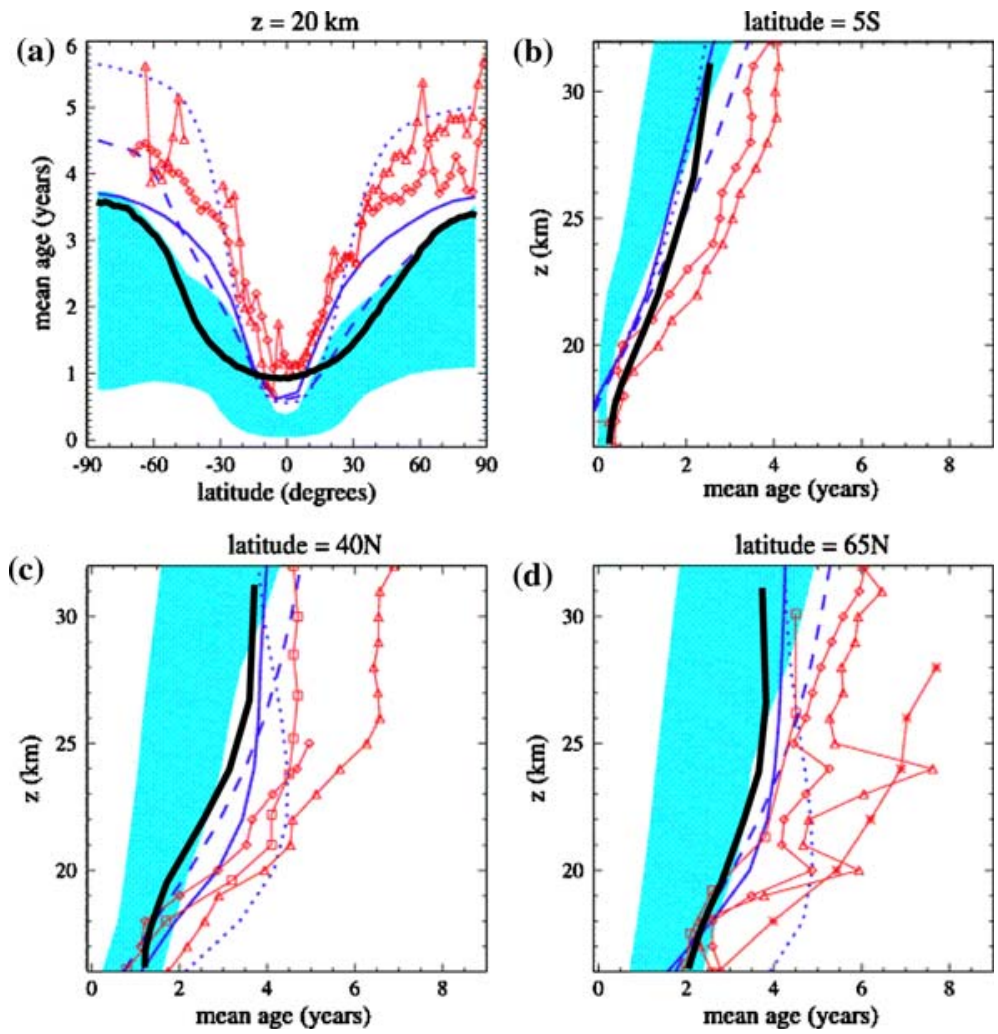

northern high latitudes, and 3-5 years at southern high latitudes. However, the observed sharp gradient in mean age in the southern hemisphere is located between $15^{\circ} \mathrm{S}$ and $30^{\circ} \mathrm{S}$, whereas in the model it is between $40^{\circ} \mathrm{S}$ and $50^{\circ} \mathrm{S}$. Thus, the observations indicate a subtropical transport barrier which is far too weak in ECHAM4.

At higher altitudes the discrepancies become more distinct. Park et al. (1999) report mean ages up to 8 years in polar vortex air above $25 \mathrm{~km}$ which is not reproduced by the model trajectory calculations, but which is closer to the modeled $\mathrm{SF}_{6}$ age of air (cf. Fig. 4). The location of the center of the top model layer at $10 \mathrm{hPa}(\approx 30 \mathrm{~km})$ might play a role here, however, Manzini and Feichter (1999)

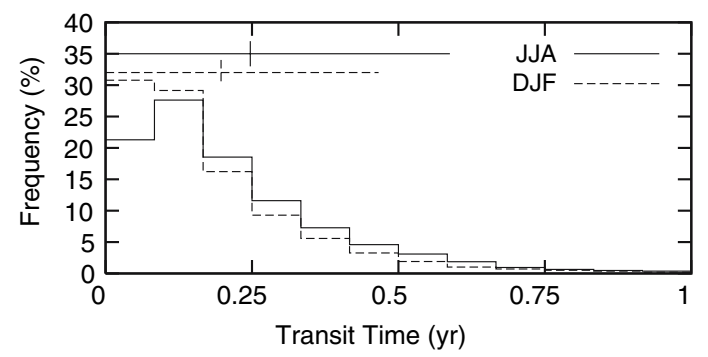

Fig. 3 Frequency distribution of transit times from the boundary layer to the tropical tropopause (equator, $100 \mathrm{hPa}$ ), averaged over boreal summer and winter. The horizontal and vertical lines indicate the mean value \pm one standard deviation. The transit times were binned into classes of one month size conducted $\mathrm{SF}_{6}$ simulations with a middle atmosphere version of ECHAM4 (semi-Lagrangian; model top layer centered at approximately $80 \mathrm{~km}$ ) and found mean ages of only 4.5 years maximum. The study of Eluszkiewicz et al. (2000) indicates that this might be caused by the numerical diffusion of the semi-Lagrangian transport scheme used in ECHAM4. Eluszkiewicz et al. calculated the mean age of air with a middle atmosphere model using a variety of advection schemes, among them the semi-Lagrangian scheme also used in ECHAM4 and two versions of a purely Lagrangian scheme. They found a strong sensitivity of the age of air to the advection scheme used, the most realistic results coming from the less diffusive schemes. This suggests that the combination of a high altitude top model level and a non-diffusive advection scheme in ECHAM4 might give more realistic results.

\subsection{Age spectra}

In order to obtain more information on the stratospheric transport in ECHAM4, we investigate now age spectra. The spectra presented in the following were derived using the "boundary layer clock", i.e., by measuring the transport times from the boundary layer to a specific location in the stratosphere.

Figure 5a shows the zonally averaged age spectra at $50 \mathrm{hPa}$ for the two seasons MAM and SON. Also shown is 

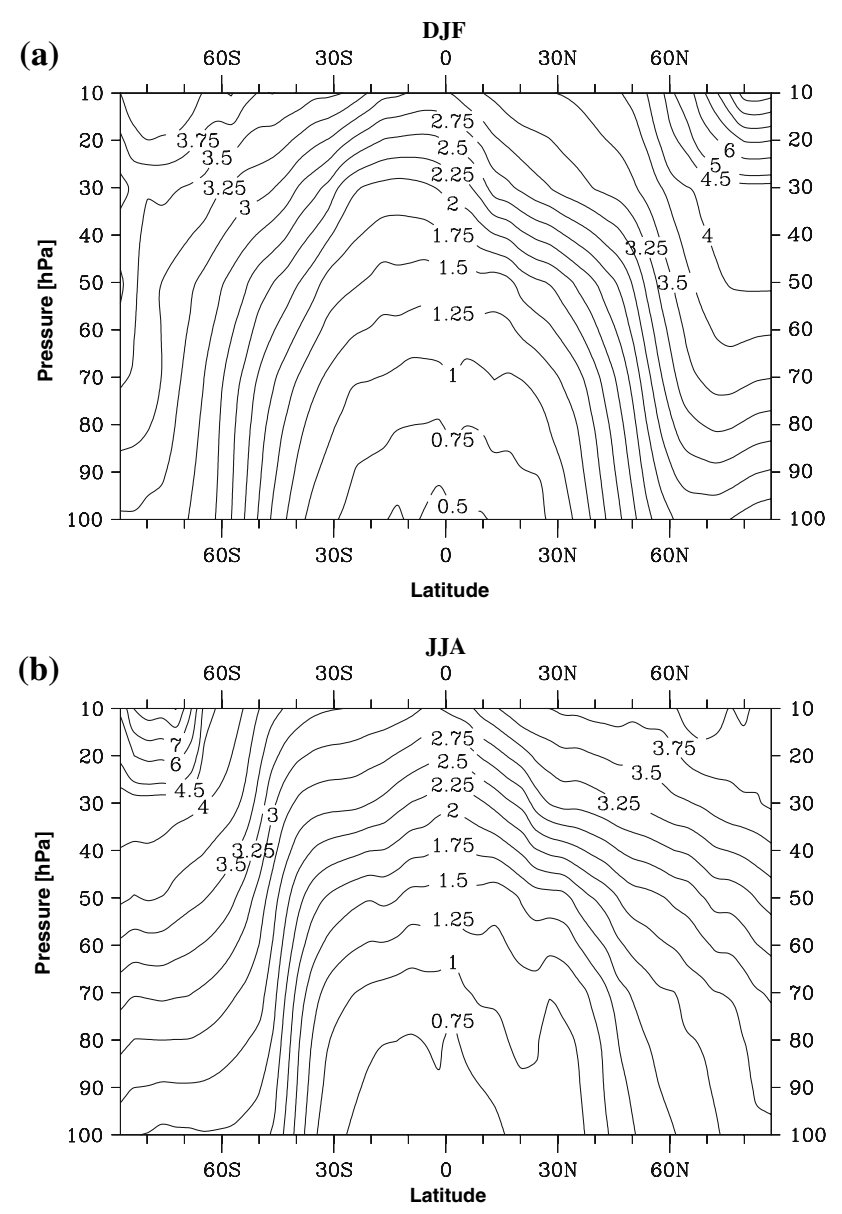

Fig. 4 Mean age of air in DJF and JJA calculated from $\mathrm{SF}_{6}$ simulation with ATTILA. Unit year

the mean age at $50 \mathrm{hPa}$ from Fig. 1. As already seen in the last section, the mean age at high latitudes is larger on the spring hemisphere than on the fall hemisphere. The formation of the polar vortex is more distinct on the southern hemisphere, and the interhemispheric difference in mean age of high latitude air is also more pronounced in austral spring.

The corresponding age spectra at low and midlatitudes exhibit the typical shape also found by many other authors (e.g., Hall and Waugh 1997; Hall et al. 1999): they are asymmetric with a peak value smaller than the mean value (which is the mean age) and a long tail towards higher values. However, approximately at the same location where the gradient in mean age is largest, the shape of the age spectrum changes from the shape described above for low latitudes to a multi-modal shape at high latitudes with several peaks which are one year apart. Since the transition is more abrupt and distinct in the southern hemisphere, we will concentrate on this hemisphere for the following investigation. Especially, we try to explain where this feature comes from, and to clarify how to interpret it correctly.
To this purpose, Fig. $5 \mathrm{~b}$ shows the temporal evolution of the age spectrum at 25 and $50 \mathrm{hPa}$, averaged over the core area of the polar vortex, from $70^{\circ} \mathrm{S}$ to $90^{\circ} \mathrm{S}$. The different maxima are clearly visible, and the shape of the age spectrum remains nearly unchanged during the course of the year (except in summer, see below), so that the individual maxima appear as lines in the figure, which are one year apart and which are sloped by one year per year (since the air is aging, trivially, by one year per year). This indicates that there is indeed little exchange with extrapolar air and that the air inside the polar vortex is relatively isolated.

The breakdown of the polar vortex and subsequent mixing with air from lower latitudes can also clearly be seen in Fig. 5b to take place in December and January: the shape of the age spectrum is changing rapidly, indicating that young air is entering polar latitudes. This air is approximately one year old (since leaving the boundary layer) at $50 \mathrm{hPa}$ and two years old at $25 \mathrm{hPa}$. It can also be seen that the period of mixing with extra-polar air is quite short (December/January). This can be explained by the dynamics in ECHAM4: as noted earlier, the polar vortex in ECHAM4 persists longer than in the observations. At $50 \mathrm{hPa}$ and at high latitudes, westerly winds even prevail throughout the whole year in ECHAM4, and at $25 \mathrm{hPa}$ easterly winds may be seen, albeit very weak, for a short time in summer only (Fig. 6).

Hence, in ECHAM4 polar stratospheric air masses have to be regarded to be relatively isolated from lower latitude air throughout the most of the year except during a short period in summer. This fact is well reflected in the age spectra of Fig. 5b, and it might also be used to explain the multiple peaks in the polar age spectra: air being isolated for one year results in shifting the age spectrum by one year without changing its shape. Mixing with extra-polar air for a short period afterwards means that the shifted spectrum is superposed with a single peak spectrum typical for lower latitude air, which results then in a multiple peak spectrum with the peaks being one year apart.

However, since the mean age of air increases with increasing height, the above explanation would imply that the peaks in the age spectra gradually move to higher values with increasing height. To test this hypothesis, Fig. $5 \mathrm{c}$ shows the variation of the polar age spectrum with altitude for the months January and July. Surprisingly, the location of the maxima are nearly constant with height. The mean age does increase with increasing height, however, not because the peaks move to higher values, but because the weights of those peaks located at higher ages are increasing. E.g., in January the first peak in the age spectrum is located at an age of 1 year, the second one at 2 years, and so on. However, the peak at 1 year is strongest between 70 and $50 \mathrm{hPa}$ and decays gradually above 

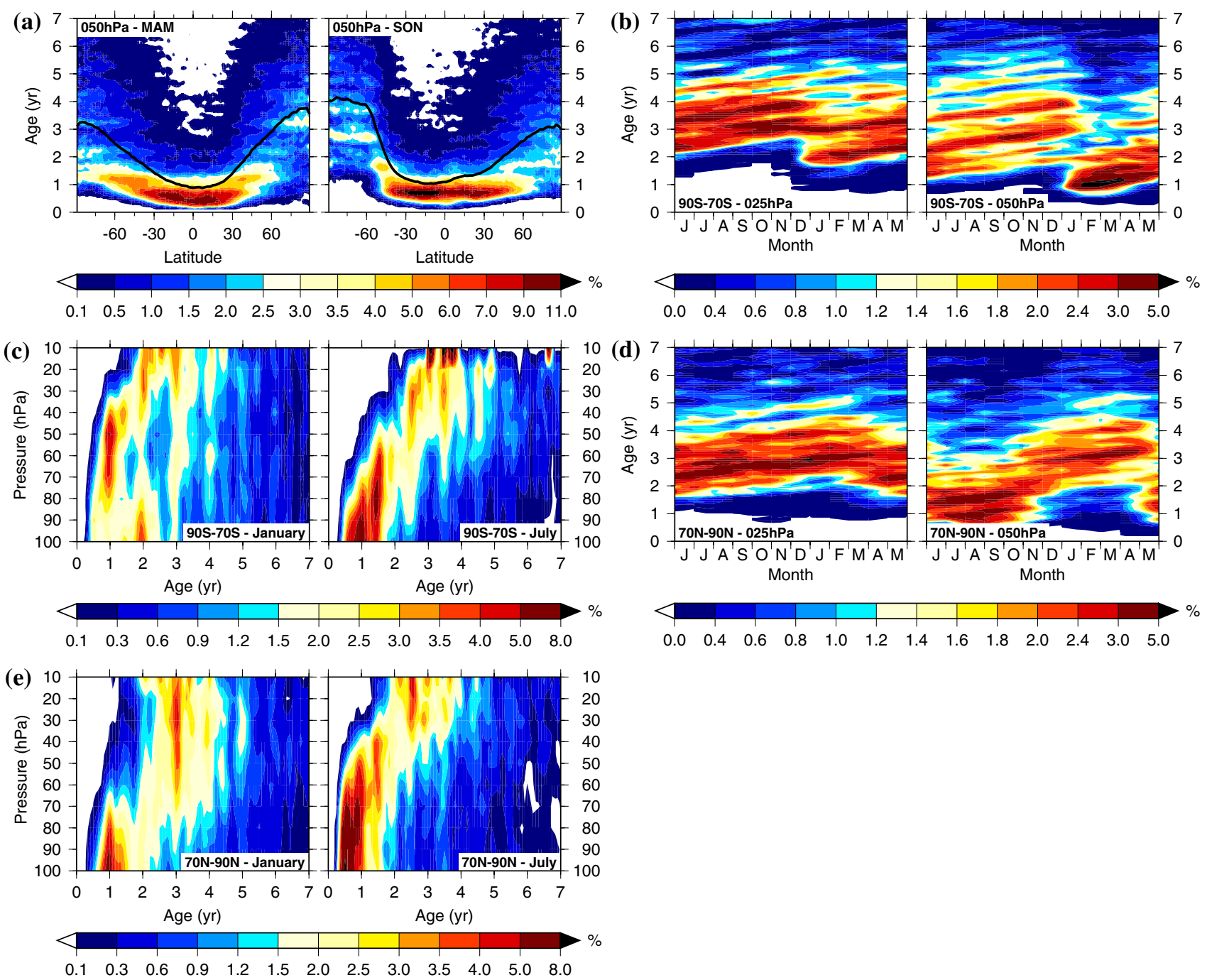

Fig. 5 a Zonally averaged age spectra at $50 \mathrm{hPa}$ in spring and fall. The age is measured as transit time since leaving the boundary layer. For each latitude the age spectrum is plotted along the $y$-axis. The black curve indicates the first moment of the spectra and corresponds to the mean age (cf. Fig. 1). b Temporal evolution of the age

$50 \mathrm{hPa}$, whereas the peaks at 2,3 , and 4 years become stronger there. Above $30 \mathrm{hPa}$ the peak at 1 year has vanished, so that in the age spectra above $30 \mathrm{hPa}$ the first peak is located at 2 years (see also Fig. 5b). These findings are in agreement with Schoeberl et al. (2003, 2005), who presented age spectra for $440 \mathrm{~K}$ potential temperature and $30 \mathrm{~km}$ height, revealing peaks one year apart.

How can this structure be explained? It obviously means that the air in the whole polar (southern) stratosphere has-independently of height-a marked preference for having entered the stratosphere in the tropics during a certain, short period of time within the year. To elucidate this fact, the trajectories at high latitudes have been analysed at 25 and $50 \mathrm{hPa}$, and have been binned according to

spectrum averaged from $70^{\circ} \mathrm{S}$ to $90^{\circ} \mathrm{S}$, at 25 and $50 \mathrm{hPa}$. c Vertical structure of the age spectrum, averaged from $70^{\circ} \mathrm{S}$ to $90^{\circ} \mathrm{S}$, for January and July. Different from $\mathbf{a}$ and $\mathbf{b}$, the age spectra are plotted along the $x$-axis. $\mathbf{d}$ Same as b but for $70^{\circ} \mathrm{N}$ to $90^{\circ} \mathrm{N}$. e Same as $\mathbf{c}$ but for $70^{\circ} \mathrm{N}$ to $90^{\circ}$

the month when they entered the stratosphere. The result is shown in Fig. 7a for the southern hemisphere. There is indeed a distinct maximum around March, which means that stratospheric trajectories at high southern latitudes have entered the stratosphere mostly in (boreal) early spring. A possible explanation for this behaviour could be the seasonal cycle of the Brewer-Dobson circulation. One might argue that the path of an air parcel through the stratosphere is strongly influenced by its time of entry into the stratosphere because of the seasonal cycle of stratospheric dynamics, and that March happens to be the time of the year when air parcels entering the stratosphere have the best chance to reach high southern latitudes later on. If this hypothesis is true, and assuming a sufficient interhemi- 

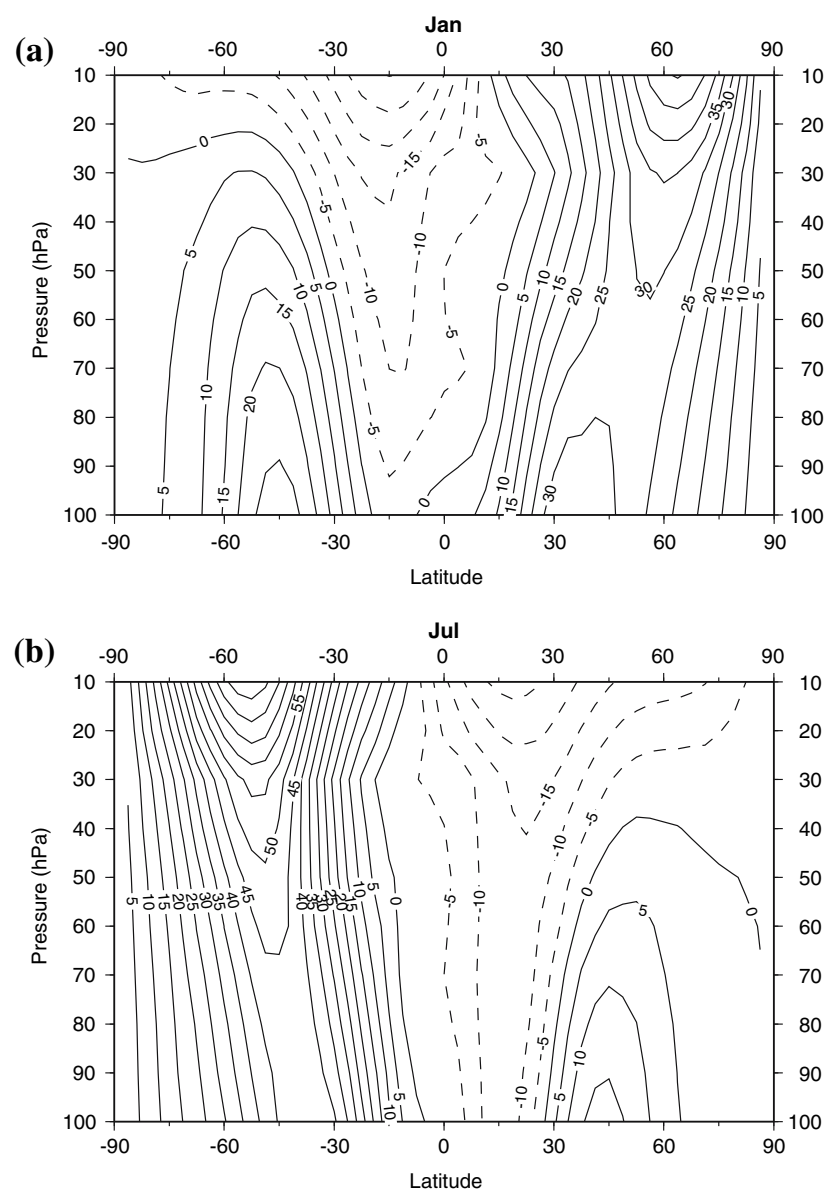

Fig. 6 Zonally and over 10 model years averaged zonal wind in January and July. Positive values mean westerlies. Unit $\mathrm{m} / \mathrm{s}$

spheric symmetry in the seasonal cycle, one will expect a similar effect on the northern hemisphere, meaning that September is the best time for air parcels to enter the stratosphere in order to have a maximum chance to reach high northern latitudes. However, Fig. 7b shows the opposite: on both hemispheres, stratospheric trajectories at high latitudes have entered the stratosphere mostly in boreal late winter and early spring. Hence, the seasonal cycle of the stratospheric circulation is no good for an explanation, even if we take into account that the seasonal cycle in the northern hemisphere is not the same as the southern hemispheric one shifted by six months.

However, Fig. 7 offers an alternative explanation, namely that there are simply more trajectories entering the stratosphere around March than in any other time of the year. Figure 8 (middle, solid line) shows for each month the number of trajectories entering the stratosphere. In order to reduce numerical noise we counted only those trajectories which stayed for at least six month in the stratosphere. There is indeed a maximum number of trajectories entering in March, and a weak secondary maximum can be seen six months later, in September, which is also apparent in Fig. 7. This correlation suggests that the seasonal cycle of the upward mass flux at the tropical tropopause is the reason for the multimodal age spectra at high latitudes. This hypothesis is corroborated by the fact that the number of trajectories entering the stratosphere which actually reach high stratospheric latitudes later on show a similar seasonal cycle (Fig. 8, middle panel, dashed line). The seasonal cycle of the upward mass flux is also reflected by the cycle of the tropical tropopause height in the model (Fig. 8, top). Fueglistaler et al. (2005) present minimum water vapour mixing ratios at $380 \mathrm{~K}$ derived from HALOE between February and March and minimum cold point temperatures between December and April, depending on the meteorological data set. Since an enhanced number of trajectories entering the stratosphere indicates enhanced ascent, adiabatic cooling and therefore lower cold point temperatures and water vapour mixing ratios, our findings agree with HALOE water vapour measurments. Scheele et al. (2005) presented a Lagrangian calculation of age of air and found larger values in the tropics for a start time in July compared to January, reflecting the seasonal cycle of the tropical ascent. However, we should note here that other authors, who investigated the meridional
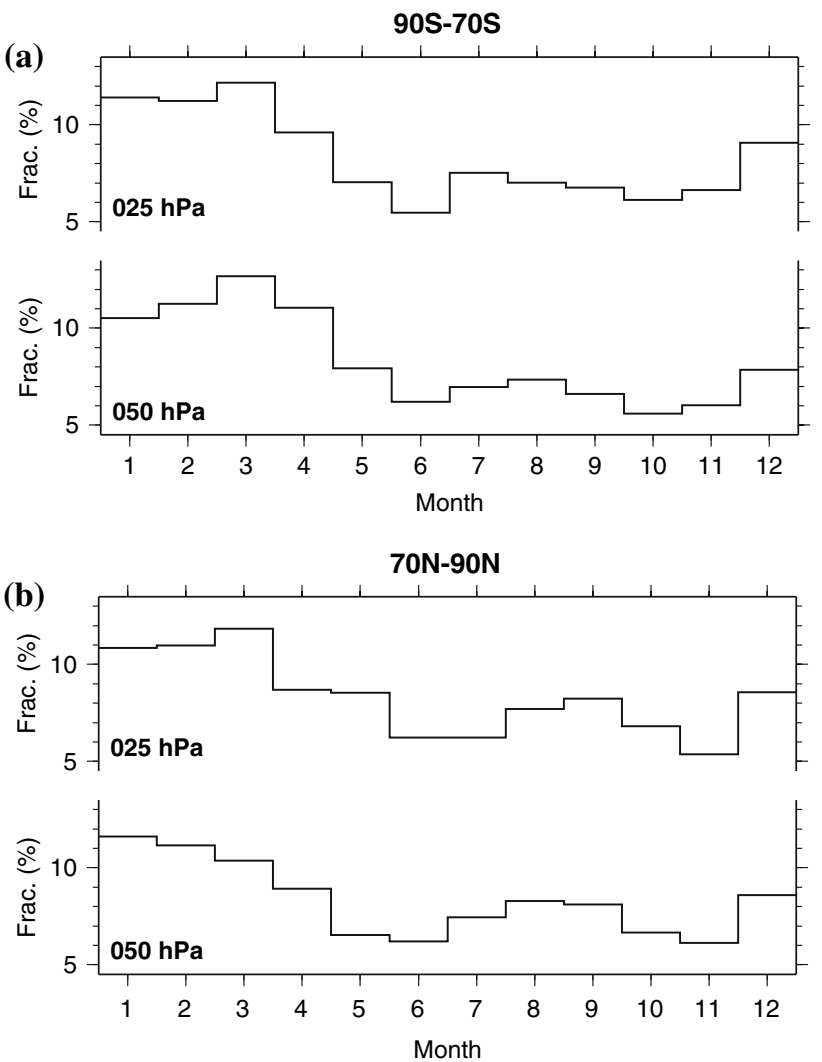

Fig. 7 a Distribution of the time points of entry into the stratosphere for trajectories poleward of $70^{\circ} \mathrm{S}$, at 50 and $25 \mathrm{hPa}$, respectively. b As a but for the northern hemisphere 

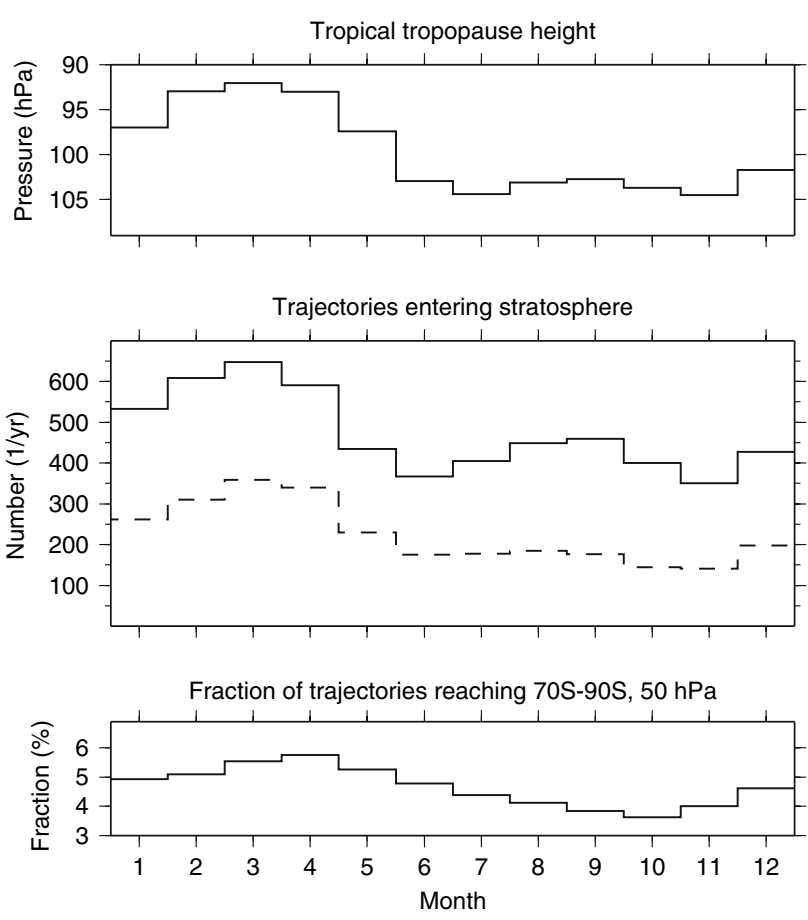

Fig. 8 Top seasonal cycle of the tropical $\left(10^{\circ} \mathrm{S}-10^{\circ} \mathrm{N}\right)$ tropopause height. Middle for each month the number of trajectories entering the stratosphere per year is shown (solid line). Only those trajectories have been counted which stayed at least 6 months in the stratosphere. Dashed line number of those trajectories entering the stratosphere which reach the southern polar vortex (defined as $70^{\circ} \mathrm{S}-90^{\circ} \mathrm{S}$ ) at $50 \mathrm{hPa}$. For easier reading this number has been multiplied by 10 . Bottom fraction of those trajectories entering the stratosphere (and staying at least 6 months) which reach the polar vortex at $50 \mathrm{hPa}$. See text for details

circulation, found the maximum of the upward flux to be in DJF, nor did they find a secondary maximum (e.g., Rosenlof 1995; Rosenlof and Holton 1993; Appenzeller et al. 1996). Latter does not necessarily disagree with our findings, since for trajectories reaching deep into the stratosphere the secondary maximum almost vanishes.

The above hypothesis also implies that if a location in the stratosphere can be reached by air parcels via several paths with different transit times, the age spectrum will have several peaks one year apart (with secondary maxima in between), because trajectories entering the stratosphere in March (and to a lesser extent in September) are overrepresented. On the other hand, if the transport path and the transit time to a certain location does not show much variation, the seasonal cycle of the upward flux at the tropical tropopause is of minor importance, and the age spectrum will have one single peak only. This can be seen in Fig. 9 which shows various age spectra at different locations in the stratosphere. In the tropics the age spectra consist mainly of one peak indicating that all air parcels reach a certain level in about the same time and via similar paths. At midlatitudes the age spectra still exhibit a main peak, however, with several secondary maxima in the tail of the spectrum. At high latitudes the age spectra show two to three equal peaks.

Basically, the northern and the southern hemisphere show the same principle pattern (Fig. 5 b,d, c, e), with a temporal shift caused by the seasonal cycle of stratospheric dynamics. In the northern hemisphere, a multi-modal age spectrum is found with a mixing time for low latitude (young) airmasses between February and April at $25 \mathrm{hPa}$ and more sharp at $50 \mathrm{hPa}$, reflecting the larger variability of the northern hemisphere vortex break down. Interestingly, the formation of the winter polar vortex is clearly visible at $50 \mathrm{hPa}$ in November. It clearly indicates the change in circulation from a summer high pressure system to winter low pressure system, which is better resolved in the northern hemisphere than in the southern hemisphere in the model (see discussion above and Fig. 6).

As an explanation for the polar age spectra, one could argue that there is no preferential transport path from the tropics to polar latitudes or that at least the variation is very large, so that the age spectrum merely reflects the seasonal cycle of the upward mass flux at the tropical tropopause. However, this does not explain the more or less abrupt transition from a single peak spectrum to a multimodal spectrum with increasing latitude, as can be seen in Fig. 5a.

We suggest that the shape of the polar age spectra is caused by a combination of two effects: first, the seasonal cycle of the upward mass flux through the tropical tropopause causes midlatitude age spectra with one main peak and secondary peaks which are one year apart. This effect also explains why the positions of the peaks are independent of the stratospheric location, a fact which can be seen in Figs. 5c and 9. The second effect has been described at the beginning of the section: polar stratospheric air masses are relatively isolated from lower latitude air throughout the most of the year except during a short period in summer (Fig. 5b). Air being isolated for one year results in shifting the age spectrum by one year without changing its shape. Subsequent mixing with extra-polar air for a short period means that the shifted spectrum is superposed with a single peak spectrum typical for midlatitude air, which results then in a spectrum with several equal peaks. This effect also explains the transition from a single peak spectrum to a multimodal spectrum with increasing latitude, because the transition zone can be interpreted as the edge of the polar vortex.

\section{Conclusions and outlook}

We have investigated the stratospheric model transport in ECHAM4 by calculating the mean age and age spectra with the Lagrangian scheme ATTILA. The Lagrangian 
Fig. 9 Age spectra (different from previous figures, measured as time since leaving the troposphere) at various locations in the stratosphere for January and July. For $25 \mathrm{hPa}$ and 50 ha around $1,500-2,000$ and 2,500 to 3,000 trajectories are taken into account, respectively (a)

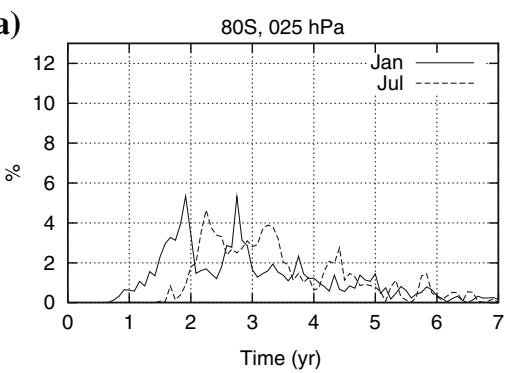

(c)

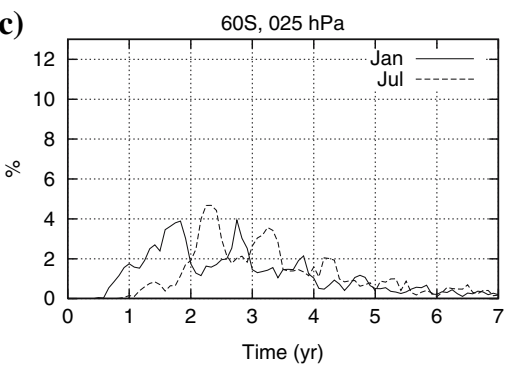

(e)
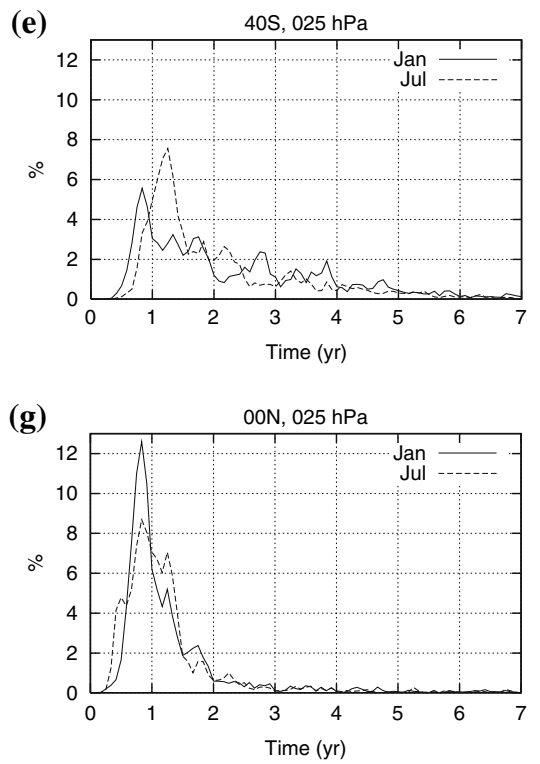

(b)

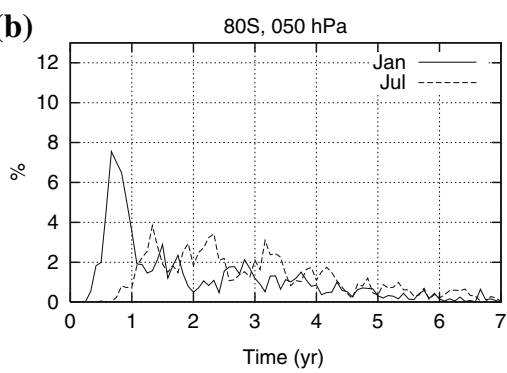

(d)

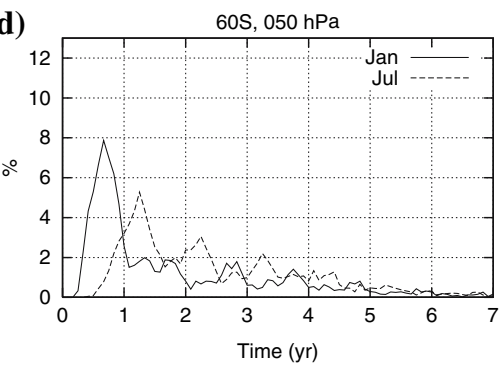

(f)

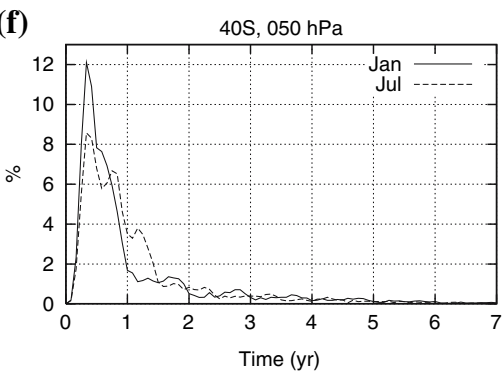

(h)

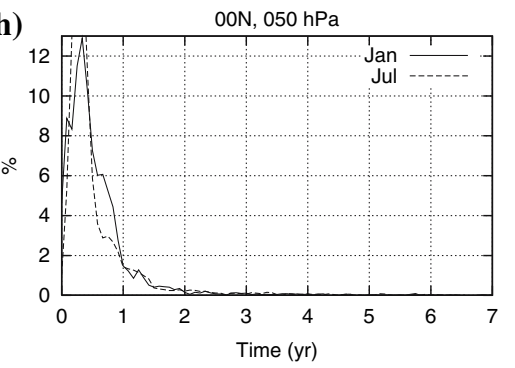

method has the advantage that it takes into account the temporal variation of the transport, and we have indeed found a strong seasonal cycle both in mean age and in the age spectra. Compared to observations, the mean age is fairly well reproduced below approximately $20 \mathrm{~km}$, however, above that level the mean age is underestimated. In addition, the strong meridional gradient in mean age on the southern hemisphere in spring, which is observed at $15^{\circ} \mathrm{S}$ to $30^{\circ} \mathrm{S}$, is located at $40^{\circ} \mathrm{S}$ to $50^{\circ} \mathrm{S}$ in the model. This indicates that the subtropical transport barrier is too weak in the model.

The edge of the polar vortex, on the other hand, acts as a very efficient transport barrier in the model. This can be seen from the temporal evolution of the polar age spectra: the polar spectra remain nearly unchanged during the most of the year (apart from being shifted to older ages), and the short period of mixing with young air from lower latitudes in summer can be clearly seen if the spectra are plotted against time.

Another feature is the variation of the shape of the age spectra with latitude. At low latitudes the spectra consist of one sharp peak, whereas at higher latitudes there are also contributions from larger transit times indicating a wider range of transport pathways. These tails in the spectra exhibit several secondary peaks which are one year apart, and at polar latitudes the age spectra even consist of several peaks of approximately equal size. We showed that the secondary peaks can be explained by the seasonal cycle of the upward mass flux at the tropical tropopause, because it causes those air parcels which entered the stratosphere 

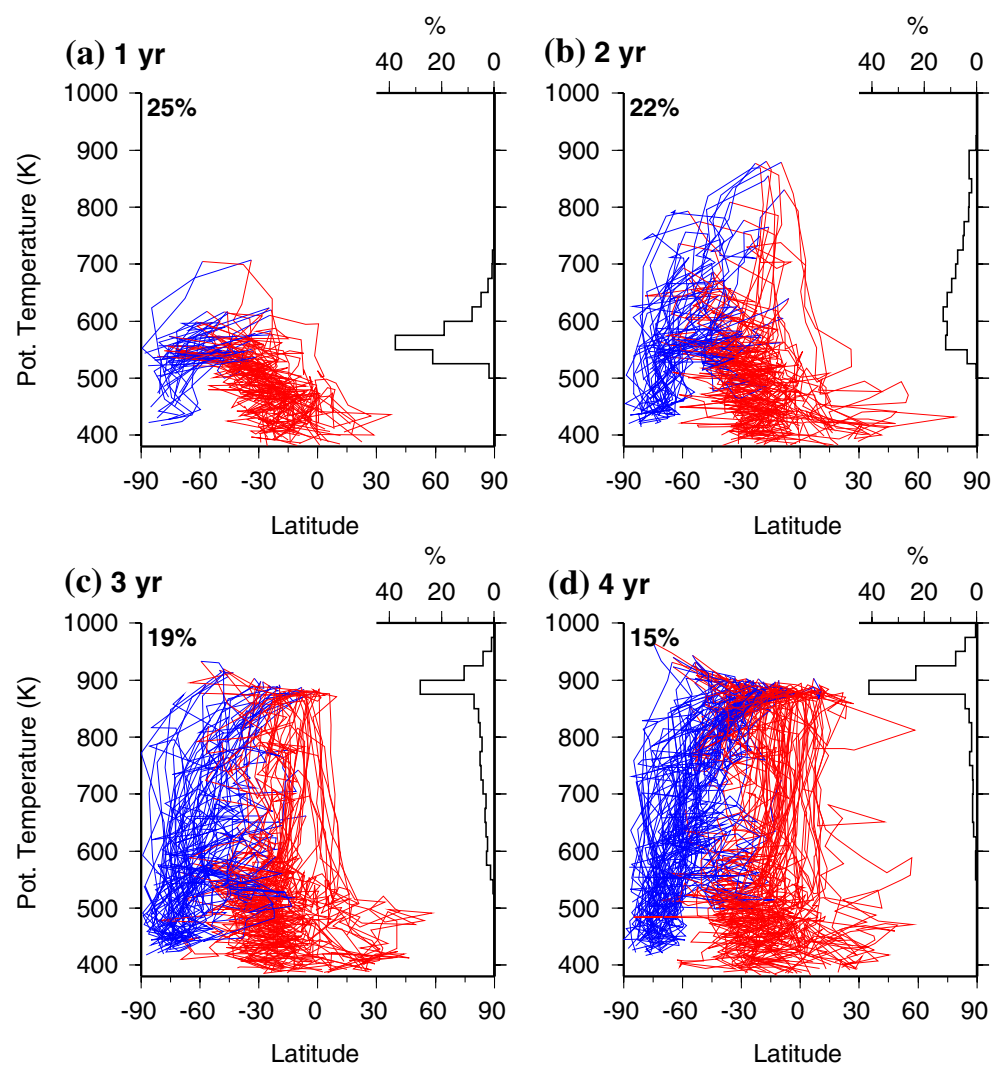

Fig. 10 a Backward trajectories are shown which start at polar latitudes $\left(70^{\circ} \mathrm{S}-90^{\circ} \mathrm{S}\right)$, at $50 \mathrm{hPa}$, and which reach the tropopause in approximately 1 year ( \pm 6 months). The figure in the left top corner indicates that these are $25 \%$ of all backward trajectories starting at $70^{\circ} \mathrm{S}-90^{\circ} \mathrm{S}, 50 \mathrm{hPa}$. For each trajectory the maximum height during its course through the stratosphere has been determined. The frequency distribution of these maximum heights is shown at the

right edge of the plot. The trajectories are plotted in red from the tropopause until reaching their maximum height and in blue from then on. For reasons of clarity only 50 randomly chosen trajectories have been plotted. b, c, d As a for trajectories which stayed in the stratosphere for 2, 3, and 4 years, respectively. There is a remaining fraction of $19 \%$ of trajectories which stayed longer than 4 years in the stratosphere and which are not shown

during the period of maximum upward flux to be stronger represented in the age spectrum. This theory also explains why the positions of the secondary peaks in the spectra are independent of the location in the stratosphere. Furthermore, we argued that the effect just described combined with the temporal evolution of the polar vortex is the reason for the multimodal age spectra at high latitudes.

In order to test these results against observations, one would need measurements of the age spectrum which in turn require measurements of a tracer with a $\delta$-like boundary condition. Such a tracer does not exist. However, Andrews et al. (1999, 2001) derived empirical age spectra from in situ observations of $\mathrm{CO}_{2}$ and $\mathrm{N}_{2} \mathrm{O}$ by assuming a reasonable shape of the age spectrum. They also found that bimodal age spectra throughout the midlatitude lower stratosphere produce time series of $\mathrm{CO}_{2}$ mixing ratio that fit observations significantly better than age spectra consisting of one single peak. The age of the older peak was also independent of altitude which agrees with our results. However, Andrews et al. (2001) calculated an age of

1 year for the younger peak and an age of 5-6 years for the older peak, whereas we found several peaks which are 1 year apart. One reason for this discrepancy might be that Andrews et al. (2001) could not test age spectra with three or more peaks, because in situ data were too scarce to adequately constrain all free parameters in the age spectra. Furthermore, the interpretation of Andrews et al. that the two peaks in the spectrum represent two predominant transport routes is not compatible with our theory, because if the seasonal cycle of the upward mass flux at the tropical tropopause is responsible for the secondary peaks in the spectrum, these peaks are also visible when there is a wide range of transport routes resp. transport times. On the other hand other modelling studies (Schoeberl et al. 2003, 2005) also found multiple peaks in the age spectra one year apart.

In order to clarify the situation and to reduce uncertainties in interpreting age spectra, one has to deal with a general problem, namely that age spectra give only information on the transport times, but not on the actual transport pathways. Hence, it would be necessary to actually 
investigate the transport pathways themselves. The easiest way to do this is to analyze trajectory data directly, which may be a topic for future studies. We would like to finish with Fig. 10 which shows backward trajectories from southern polar latitudes at $50 \mathrm{hPa}$. The distribution of the maximum heights attained by each trajectory during its course through the stratosphere has also been determined, and each trajectory is plotted in different colours before and after reaching its maximum height.

The trajectories have been binned into four classes depending on how long they needed to travel from the tropics to polar latitudes. Figure 10a (travel time 1 year) and 1,000 days (travel time 4 years) indeed indicate that there are two predominant transport routes as suggested by Andrews et al. (2001). (For observational evidence for this short pathway see for example Fu et al. 2006). However, the trajectories which travel 2-3 years in the stratosphere are approximately as numerous as the trajectories travelling 1 and 4 years, and do not exhibit a clear structure. At a first glance one might conclude from Fig. $10 \mathrm{~b}$ to $\mathrm{d}$ that meridional transport from the tropics to midlatitudes takes place mainly below $600 \mathrm{~K}$ and above $750 \mathrm{~K}$ with a minimum at around $700 \mathrm{~K}$. This is in agreement with the studies of Chen et al. (1994) and Waugh (1996), however, the trajectories shown in Fig. 10 are backward trajectories starting at $50 \mathrm{hPa}$ only, so that a closer examination of the data will be needed to corroborate this conclusion.

Hall (2000) presented a similar analysis, showing a mean increase in maximum height of the pathways to $22 \mathrm{~km}$ and $44^{\circ} \mathrm{N}$, from around 25 to $32 \mathrm{~km}$ for 1 to 4 years travelling time. Although different starting and endpoints are considered a comparable behaviour is found in both models, except for those trajectories travelling through the upper stratosphere, which are not represented in ECHAM4 due to the low vertical resolution.

What can be seen clearly from Fig. 10c and d is the fact that those trajectories which need 3 years and longer to travel from the tropics to polar latitudes ascend in the tropics to heights mostly above $850 \mathrm{~K}$ and move poleward after reaching their maximum height while descending. However, the sharp gradient in the distribution of the maximum heights of those trajectories and the fact that the centre of the uppermost model layer is located somewhat lower than $900 \mathrm{~K}$ in the tropics indicates that it somehow misrepresents transport routes via higher levels which exist in the real atmosphere and which contribute to the observed high mean age of polar vortex air. However, the upper branch of the Brewer-Dobson circulation is mainly confined to the uppermost model layer, which leads to reasonable pattern in the meridional circulation. As a consequence, the transport to the winter hemisphere is too fast and age of air is reduced (a discussion of the resolution in the upper model domain on dynamics and circulation is given in Grewe 2006). Hence, the coarse resolution in the upper model domain is certainly a limitation to the model results. However the principal results are not considered to be significantly affected. Nevertheless, we think that a further direct investigation of 3D trajectories may produce valuable information on stratospheric dynamics and transport.

Acknowledgments The authos thank Dr. C. Johnson (UK Met. Office) for generously providing the STOCHEM code, which facilitated the development of ATTILA considerably and two anonymous reviewer for fruitful comments. This work was supported by the AFS project of the German Bundesministerium für Bildung und Forschung (project 07 AF $311 / 3$ ).

\section{References}

Andrews AE, Boering KA, Daube BC, Wofsy SC, Hintsa EJ, Weinstock EM, Bui TP (1999) Empirical age spectra for the lower tropical stratosphere from in situ observations of $\mathrm{CO}_{2}$ : implications for stratospheric transport. J Geophys Res 104(D21):26581-26595

Andrews AE, Boering KA, Wofsy SC, Daube BC, Jones DB, Alex S, Loewenstein M, Podolske JR, Strahan SE (2001) Empirical age spectra for the midlatitude lower stratosphere from in situ observations of $\mathrm{CO}_{2}$ : quantitative evidence for a subtropical "barrier" to horizontal transport. J Geophys Res 106(D10):10257-10274

Appenzeller C, Holton JR, Rosenlof KH (1996) Seasonal variation of mass transport across the tropopause. J Geophys Res 101(D10):15071-15078

Bonazzola M, Haynes PH (2004) A trajectory-based study of the tropical tropopause region. J Geophys Res 109:D20112 doi:10.1029/2003JD004356

Brinkop S, Sausen R (1997) A finite difference approximation for convective transports which maintains positive tracer concentrations. Beitr Phys Atmos 70(3):245-248

Chen P, Holton JR, O’Neill A, Swinbank R (1994) Isentropic mass exchange between the tropics and extratropics in the stratosphere. J Atmos Sci 51(20):3006-3018

Eluszkiewicz J, Hemler RS, Mahlman JD, Bruhwiler L, Takacs LL (2000) Sensitivity of age-of-air calculations to the choice of advection scheme. J Atmos Sci 57(19):3185-3201

Eyring V, Dameris M, Grewe V, Langbein I, Kouker W (2003) Climatologies of subtropical mixing derived from 3D models. Atmos Chem Phys 3:1007-1021

Fu R, Hu Y, Wright JS, Jiang JH, Dickinson RE, Chen M, Filipiak M, Read WG, Waters JW, Wu DL (2006) Short circuit of water vapour and polluted air to the global stratosphere by convective transport over the Tibetian Plateau. Proc Natl Acad Sci USA 103, 5664-5669 doi:10.1073/pnas.0601584103

Fueglistaler S, Haynes PH (2005) Control of interannual and longerterm variability of stratospheric water vapor. J Geophys Res 110:D24108 doi:10.1029/2005JD006019

Fueglistaler S, Wernli H, Peter T (2004) Tropical tropospheretostratosphere transport inferred from trajectory calculations. J Geophys Res 109:D03108, doi:10.1029/ 2003JD004069

Fueglistaler S, Bonazzola M, Haynes PH, Peter T (2005) Stratospheric water vapor predicted from the Lagrangian temperature history of air entering the stratosphere in the tropics. J Geophys Res 110:D08107, doi:10.1029/2004JD005516

Grewe V (2006) The origin of ozone. Atmos Chem Phys 6:1495-1511 
Grewe V, Stenke A, Ponater M, Sausen R, Pitari G, Iachetti D, Rogers H, Dessens O, Pyle J, Isaksen ISA, Gulstad L, Søvde OA, Marizy C, Pascuillo E (2007) Climate impact of supersonic air traffic: an approach to optimize a potential future supersonic fleet? Results from the EUproject SCENIC. Atmos Chem Phys Discuss 7:6143-6187

Hall TM (2000) Path histories and timescales in stratospheric transport: analysis of an idealized model. J Geophys Res 105(D18):22811-22823

Hall TM, Plumb RA (1994) Age as a diagnostic of stratospheric transport. J Geophys Res 99(D1):1059-1070

Hall TM, Waugh DW (1997) Timescales for the stratospheric circulation derived from tracers. J Geophys Res 102(D7):8991-9001

Hall TM, Waugh DW, Boering KA, Plumb RA (1999) Evaluation of transport in stratospheric models. J Geophys Res 104(D15): 18815-18839

Hatsushika H, Yamazaki K (2003) Stratospheric drain over Indonesia and dehydration within the tropical tropopause layer diagnosed by air parcel trajectories. J Geophys Res 108(D19):4610 doi:10.1029/2002JD002986

Hein R, Dameris M, Schnadt C, Land C, Grewe V, Köhler I, Ponater M, Sausen R, Steil B, Landgraf J, Brühl C (2001) Results of an interactively coupled atmospheric chemistry-general circulation model: comparison with observations. Ann Geophysicae 19:435-457

Holton JR, Haynes PH, McIntyre ME, Douglas AR, Rood RB, Pfister L (1995) Stratosphere-troposphere-exchange. Rev Geophys 33:403-439

Kida H (1983) General circulation of air parcels and transport characteristics derived from a hemispheric GCM, Part 2. Very long-term motions of air parcels in the troposphere and stratosphere. J Met Soc 61(4):510-522

Lelieveld J, Brühl C, Jöckel P, Steil B, Crutzen PJ, Fischer H, Giorgetta MA, Hoor P, Lawrence MG, Milz M, Sausen R, Stiller GP, Tost H (2006) Stratospheric dryness. Atmos Chem Phys Disc 6:11247-11298

Manzini E, Feichter J (1999) Simulation of the SF6 Tracer with the middle atmosphere MAECHAM4 Model: aspects of the largescale transport. J Geophys Res 104(D24):31097-31108

Park JH, Ko MKW, Jackman CH, Plumb RA, Kaye JA, Sage KH (eds) (1999) Models and measurements intercomparison II, NASA Tech. Publ., NASA/TM-1999-209554, National Aeronautics and Space Administration, $494 \mathrm{pp}$

Press WH, Flannery BP, Teukolsky SA, Vetterling WT (1990) Numerical Recipes-the art of scientific computing (FORTRAN Version). Cambridge University Press, Cambridge

Rasch PJ, Williamson DL (1990) Computational aspects of moisture transport in global models of the atmosphere. Q J R Met Soc 116:1071-1090

Ray EA, Moore FL, Elkins JW, Dutton GS, Fahey DW, Vömel H, Oltmans SJ, Rosenlof KH (1999) Transport into the Northern Hemisphere lowermost stratosphere revealed by in situ tracer measurements. J Geophys Res 104(D21):26565-26580

Reddmann T, Ruhnke R, Kouker W (2001) Three-dimensional model simulations of SF6 with mesospheric chemistry. J Geophys Res 106(D13):14525-14537
Reithmeier C (2001) Untersuchungen zum globalen Spurenstofftransport und Stratosphären-Troposphären-Austausch mit dem Lagrangeschen Modell ECHAM4/ATTILA, Ph.D Thesis, May 2001, Ludwig-Maximilians-Universität, München, available as: Forschungsbericht 2001-09, ISSN 1434-8454, Deutsches Zentrum für Luft- und Raumfahrt eV (DLR), Köln

Reithmeier C, Sausen R (2002) ATTILA-atmospheric tracer transport in a Lagrangian model. Tellus 54(3):278-299

Roeckner E, Arpe K, Bengtsson L, Brinkop S, Dümenil L, Esch M, Kirk E, Lunkeit F, Ponater M, Rockel B, Sausen R, Schlese U, Schubert S, Windelband M (1992) Simulation of the present-day climate with the ECHAM model: impact of model physics and resolution, report no. 93, ISSN 0937-1060, Max-Planck-Institut für Meteorologie, Hamburg

Roeckner E, Arpe K, Bengtsson L, Christoph M, Claussen M, Dümenil L, Esch M, Giorgetta M, Schlese U, Schulzweida U (1996) The atmospheric general circulation model ECHAM-4: model description and simulation of present-day climate, report no. 218, ISSN 0937-1060, Max-Planck-Institut für Meteorologie, Hamburg

Rosenlof KH (1995) Seasonal cycle of the residual mean meridional circulation in the stratosphere. J Geophys Res 100(D3):51735191

Rosenlof KH, Holton JR (1993) Estimates of the stratospheric residual circulation using the downward control principle. J Geophys Res 98(D6):10465-10479

Scheele MP, Siegmund PC, van Velthoven PFJ (2005) Stratospheric age of air computed with trajectories based on various 3D-Var and 4D-Var data sets. Atmos Chem Phys 5:1-7

Schoeberl MR, Sparling LC, Jackman CH, Fleming EL (2000) A Lagrangian view of stratospheric trace gas distributions. J Geophys Res 105(D1):1537-1552

Schoeberl MR, Douglass AR, Zhu Z, Pawson S (2003) A comparison of the lower stratospheric age spectra derived from a general circulation model and two data assimilation systems. J Geophys Res 108(D3):4113, doi:10.1029/2002JD002652

Schoeberl MR, Douglass AR, Polansky B, Boone C, Walker KA, Bernath P (2005) Estimation of stratospheric age spectrum from chemical tracers. J Geophys Res 110:D21303, doi:10.1029/ 2005JD006125

Stenke A, Grewe V, Ponater M (2007) Lagrangian transport of water vapor and cloud water in the ECHAM4 GCM and its impact on the cold bias. Clim Dyn (revised)

Volk CM, Elkins JW, Fahey DW, Dutton GS, Gilligan JM, Loewenstein M, Podolske JR, Chan KR, Gunson MR (1997) Evaluation of source gas lifetimes from stratospheric observations. J Geophys Res 102(D21):25543-25564

Waugh DW (1996) Seasonal variation of isentropic transport out of the tropical stratosphere. J Geophys Res 101(D2):4007-4023

Waugh DW, Hall TM (2002) Age of stratospheric air: theory, observations, and models. Rev Geophys 40(4):1010 doi:10.1029/ 2000RG000101

Williamson DL, Rasch PJ (1994) Water vapor transport in the NCAR CCM2. Tellus 46A:34-51 